\section{Posterior reversible encephalopathy syndrome in a B-cell acute lymphoblastic leukemia young adult patient treated with a pediatric-like chemotherapeutic schedule}

Cristina Papayannidis, Francesca Volpato, Ilaria lacobucci, Maria Chiara Abbenante, Chiara Sartor, Giovanni Martinelli

Department of Experimental, Diagnostic and Specialty Medicine, Bologna

University, Italy

\section{Abstract}

We report here the case of a young adult affected by pre B-cell acute lymphoblastic leukemia (ALL), who developed, during a pediatric-like chemotherapy consolidation schedule with high dosage of Methotrexate, a severe neurological toxicity. Clinical presentation and neuroimaging data were diagnostic for posterior reversible encephalopathy syndrome (PRES). A complete resolution was quickly obtained with medical blood pressure control and anticonvulsants administration. To the best of our knowledge, this is the first case of PRES described in the adult ALL setting. Currently, the clinical management of this aggressive disease is moving towards a pediatric-like approach also in adult patients, due to the better outcome reached with intensive chemotherapeutic regimens in children population. However, therapy-related toxicities have to be taken into account, since their onset may adversely affect patients' clinical outcome.

\section{Case Report}

An 18-year-old boy was admitted to our hospital (Bologna, Italy) due to onset of fatigue and persistent fever, unresponsive to antibacterial therapy. Blood analyses showed a white blood cell count of $93,850 / \mu \mathrm{L}$, with $99 \%$ of lymphoid blast cells, hemoglobin of $11 \mathrm{~g} / \mathrm{dL}$, and a platelet count of $70,000 / \mu \mathrm{L}$. The bone marrow evaluation was diagnostic for a pre-B cell acute lymphoblastic leukemia (ALL), CD34, CD19 and CD22 positive at the immunophenotype analysis, with normal karyotype and without central nervous system (CNS) involvement.

According to institutional guidelines and after informed consent was signed, the patient received a chemotherapeutic program, based on the AIEOP LAL2000 clinical protocol back- bone, including a steroid pre-treatment, an induction phase 1A (Vincristine, Daunorubicin, L-Asparaginase, Prednisone and intrathecal CNS prophylaxis) and a phase 1B (Cyclophosphamide, oral 6-Mercaptopurine and Cytarabine). After this treatment, the patient obtained a complete morphologic remission, with the persistence of positive minimal residual disease, evaluated by polymerase chain reaction rearrangement of immunoglobulin $\mathrm{H}$ gene on day 33 , which became negative on day 78 .

Thus, the patient underwent a third cycle of chemotherapy, which included high dose of Methotrexate (MTX) $\left(3 \mathrm{~g} / \mathrm{m}^{2}\right)$ followed by Cyclophosphamide (400 mg), Cytarabine (3900 mg), Vincristine (2 mg) and intrathecal MTX (10 mg). This therapy was well tolerated, and no adverse events occurred. Therefore, after hematologic recovery was reached, the second dosage of HD-MTX was administered, followed by Vindesine ( $5 \mathrm{mg}$ ), Ifosfamide (3200 mg) and Daunorubicin (50 mg).

Nine days after MTX infusion, during the iatrogenic aplasia phase, the patient suddenly developed generalized tonic clonic seizures, with loss of consciousness. His blood pressure was 160/100 $\mathrm{mmHg}$; he was confused, and a detailed neurologic examination showed a strong-force defect involving the left arm. Coagulation investigation was normal. Promptly, Diazepam and Dexametasone were administered, with a momentaneus improvement of his clinical condition.

Despite the resolution of this event, the
Correspondence: Cristina Papayannidis, Department of Experimental, Diagnostic and Specialty Medicine, Bologna University, via Massarenti 9, 40138 Bologna, Italy.

Tel.: +39.051.636.3973 - Fax: +39.051.636.4037.

E-mail: cristina.papayannidis@unibo.it

Key words: posterior reversible encephalopathy syndrome, acute lymphoblastic leukemia, methotrexate, seizure, brain magnetic resonance imaging.

Contributions: the authors contributed equally.

Conflict of interests: the authors declare no potential conflict of interest.

Received for publication: 21 July 2014. Accepted for publication: 21 July 2014.

This work is licensed under a Creative Commons Attribution NonCommercial 3.0 License (CC BYNC 3.0).

(C) Copyright C. Papayannidis et al., 2014

Licensee PAGEPress, Italy

Hematology Reports 2014; 6:5565

doi:10.4081/hr.2014.5565

patient showed two further episodes of epilepsy during the same day. Therefore, a brain computed tomography (CT) was immediately performed, revealing the presence of hypodense areas in the bilateral frontal lobes and right parietal parasagittal region, with no
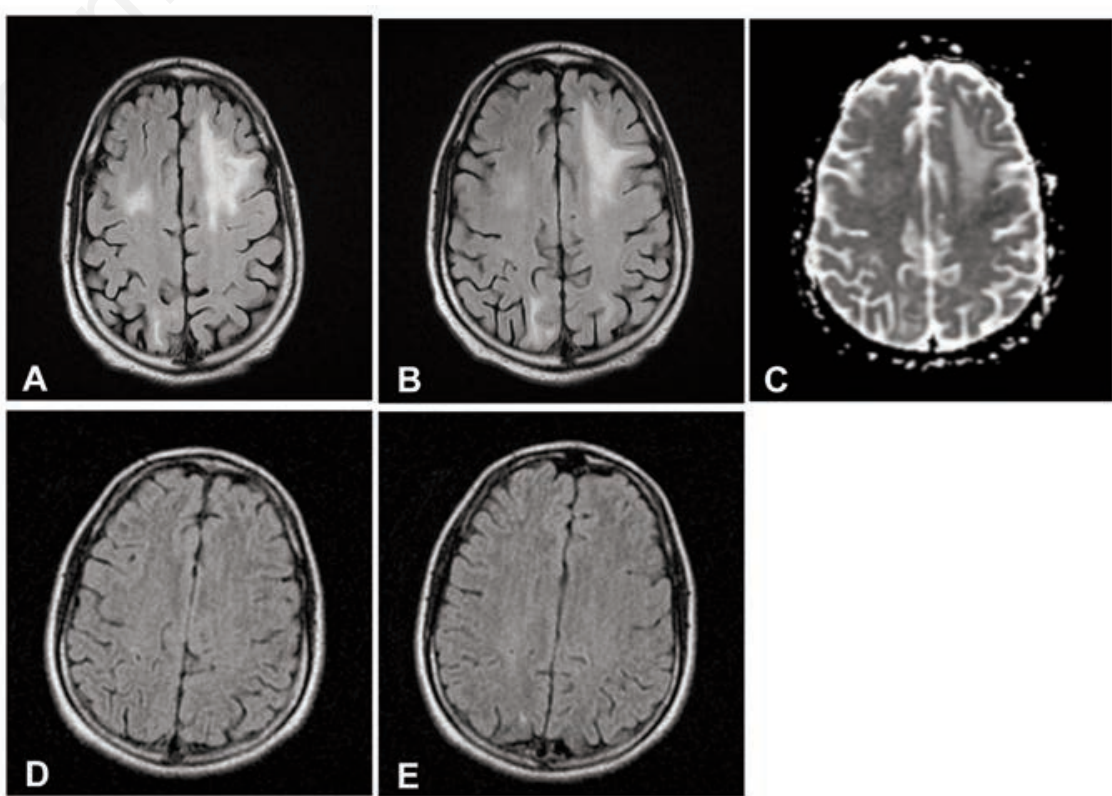

Figure 1. A,B) Brain magnetic resonance imaging (MRI) imaging (Flair T2 sequence), showing hyperintense lesions in the subcortical white matter of frontal lobes and right parietal parasagittal region consistent with vasogenic edema. C) Apparent diffusion coefficient map showing elevated signal intensities in these lesions. D,E) MRI imaging nine months later, showing almost complete resolution of the lesions. 
intracranial bleeding or venous thrombosis. Consistently, MRI fluid-attenuated inversion recovery and diffusion-weighted imaging revealed subcortical and cortical hyperintensities in the bilateral frontal lobes and parietal parasagittal region (Figure 1A,B). In addiction, the apparent diffusion coefficient (ADC) map showed elevated signal intensities in the bilateral frontal lobes (Figure 1C). These MRI observations were suggestive of vasogenic edema rather than cytotoxic edema. In order to exclude CNS infective involvement, a lumbar puncture was performed, which resulted negative for microbiological agents and leukemic cells. Therefore, based on neuroimaging results and clinical presentation, a diagnosis of posterior reversible encephalopathy syndrome (PRES) was made.

The patient was admitted to the Intensive Care Unit for monitoring and seizure treatment. A prophylactic therapy with phenytoin and anti-hypertensive drugs was introduced, and a progressive improvement of clinical conditions, neurological symptoms and cognitive status was observed. The patient reached a complete hematological recovery, but due to the severe neurological complication, his chemotherapeutic program was prematurely stopped. Seven months later, a brain MRI demonstrated a complete resolution of previous detected lesions (Figure 1D,E). Unfortunately, the patient relapsed eight months later and died as a consequence of progression disease.

\section{Discussion and Conclusions}

Posterior reversible encephalopathy syndrome is a clinical-neuroradiological entity, firstly identified by Hinchey et al. in 1996. This condition is often associated with different diseases such as cancer, hypertension, autoimmune abnormalities, eclampsia, sepsis, renal disorders, drugs and bone marrow and solid organ transplant. The clinical presentation is characterized by seizure, which is the most common symptom, headache, confusion, decreased level of consciousness and coma. Visual disturbances include hemianopia, visual neglect and cortical blindness. At neu- roimaging, PRES is characterized by transient bilateral lesions, that predominantly affect white matter of the occipital-parietal lobes and can be identified by MRI scan, that allows a more accurate etiological diagnosis compared to CT. PRES management includes withdrawal of damaging agents, blood pressure control and anticonvulsants in patients with seizures. Previously, neurotoxicity has been observed after the administration of high-dose and/or intrathecal MTX in the pediatric population; conversely, little is known in adult patients. To the best of our knowledge, this is the first report of a pre-B ALL young adult patient treated with high dose of MTX, who developed a PRES.

Currently, in ALL, there is a trend towards more intensive treatment of adult patients with pediatric-inspired regimens, based on the high remission rates obtained by this kind of approach. However, an accurate knowledge of potential therapy related toxicities, including neurologic adverse events, is required for the safe and correct management of these patients. 